\title{
A 3D view of galactic winds in luminous infrared galaxies
}

\section{P. Martín-Fernández ${ }^{1}$ J. Jiménez-Vicente ${ }^{1,2}$, A. Zurita ${ }^{1,2}$, E. Mediavilla ${ }^{3,4}$ and A. Castillo-Morales ${ }^{5}$}

${ }^{1}$ Dpto. Física Teórica y del Cosmos, Universidad de Granada, 18071-Granada, Spain

${ }^{2}$ Instituto Carlos I de Física Teórica y Computacional, Granada, Spain

${ }^{3}$ Instituto de Astrofísica de Canarias, 38200-La Laguna, Sta. Cruz de Tenerife, Spain

${ }^{4}$ Dpto. de Astrofísica, Universidad de La Laguna, 38200-La Laguna, Tenerife, Spain

${ }^{5}$ Dpto. de Astrofísica y CC. de la Atmósfera, UCM, 28040-Madrid, Spain

email: pablomartin@ugr.es

\begin{abstract}
Galactic winds and outflows are an ubiquitous phenomenon in galaxies with active star formation and/or active nuclei. They constitute the main mechanism for redistributing dust and metals on large scales and are therefore a key ingredient to understand the life cycle of galaxies. Among galaxies, ULIRGs are of particular interest in this context, as they host intense starbursts and are likely to be the dominant star formers at $\mathrm{z}>1$. These objects have been shown to host important winds, but it is not yet known what is the frequency of galactic winds and their properties in galaxies with lower star formation rates (SFR). We are studying galactic winds in a sample of 21 galaxies with different SFRs (including ULIRGs) from observations with the INTEGRAL fiber spectrograph on the $4.2 \mathrm{~m}$ WHT. In order to be able to address the complex multi-phase nature of the wind phenomenon, we have used the Na I D doublet absorption lines to trace cold gas, and a few emission lines ( $\mathrm{H} \alpha$, [N II] and $[\mathrm{S}$ II] $)$ to trace the warmer ionized gas of the wind. The distribution and kinematics of both components in these objects is then analysed. Preliminary results show strong spatial correlation between regions with high non-circular velocities, areas with high star formation activity and regions with two different components in the emission lines. This set of data will help us to characterise the distribution and kinematics of the winds and their relation with the host galaxy type.
\end{abstract}

Keywords. galaxies: spirals - galaxies: evolution - ISM: jets and outflows

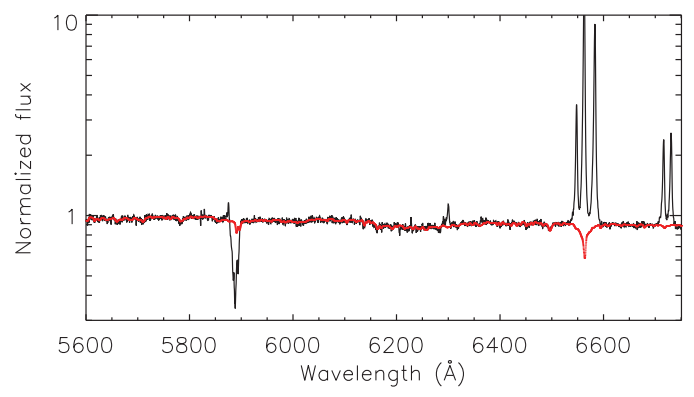

Figure 1. Integrated spectrum of NGC 5394 (solid black) with overlaid fit to the stellar population spectrum (dotted red). In this case, most of the Na I D absorption is produced in the interstellar medium. The subtraction of the synthetic stellar spectra from the total observed spectra yields a spectrum of the nebular component alone for subsequent kinematic analysis.

We acknowledge support from the Spanish "Ministerio de Economía y Competitividad" and "Junta de Andalucía" via grants AYA2011-24728 and FQM-108. 